\title{
Application of Different $H(x)$ in Homotopy Analysis Methods for Solving Systems of Linear Equations
}

\author{
Mohammad Hasan Khani' ${ }^{1}$ Jalil Rashidinia ${ }^{2}$, Sajjad Zia Borujeni1,2* \\ ${ }^{1}$ Department of Mathematics, Islamic Azad University, Shahin Shahr Branch, Isfahan, Iran \\ ${ }^{2}$ Department of Mathematics, Islamic Azad University, Central Tehran Branch, Tehran, Iran \\ Email: Azaliyazia@yahoo.com, ${ }^{*}$ Saj.ziaBorujeni.sci@iauctb.ac.ir
}

Received 6 August 2015; accepted 18 September 2015; published 21 September 2015

Copyright (C) 2015 by authors and Scientific Research Publishing Inc.

This work is licensed under the Creative Commons Attribution International License (CC BY). http://creativecommons.org/licenses/by/4.0/

c) (i) Open Access

\begin{abstract}
In this paper, we present homotopy analysis method (HAM) for solving system of linear equations and use of different $H(x)$ in this method. The numerical results indicate that this method performs better than the homotopy perturbation method (HPM) for solving linear systems.
\end{abstract}

\section{Keywords}

HAM, HPM, Linear System

\section{Introduction}

Approximating the solutions of the system of linear and nonlinear equations has widespread applications in applied mathematics [1]-[11]. Many techniques including homotopy perturbation method (HPM) [12] and iterative methods [13] were suggested to search for the solution of linear systems. In 2009 Keramati [2] and in 2011 Liu [3] in their articles applied HPM to the solution of the system $A x=b$. In this article we used homotopy analysis method [14] [15] with different $H(x)$ to solve linear system $A x=b$ and showed that our results were better than the HPM results; then convergence of the method was considered.

Consider a linear system

$$
A x=b,
$$

where $A=\left[a_{i j}\right] \in R^{n \times n}$ is nonsingular and $x, b \in R^{n}$ is a vector.

First of all, the basic ideas of the homotopy analysis method are being discussed.

\footnotetext{
${ }^{*}$ Corresponding author.
}

How to cite this paper: Khani, M.H., Rashidinia, J. and Zia Borujeni, S. (2015) Application of Different $H(x)$ in Homotopy Analysis Methods for Solving Systems of Linear Equations. Advances in Linear Algebra \& Matrix Theory, 5, 129-137. http://dx.doi.org/10.4236/alamt.2015.53012 
Let $x_{0}$ be an initial guess of $x$, and $q \in[0,1]$ be called the embedding parameter. The homotopy analysis method is based on a kind of continuous mapping $x \rightarrow \phi(x ; q)$ such that, as the embedding parameter $q$ increases from 0 to $1, \phi(x ; q)$ varies from the initial guess $x_{0}$ to the exact solution $x$. To ensure this, choose such an auxiliary linear operator as

$$
L(\phi(x, q))=\phi(x, q)
$$

and we define the operator

$$
N(\phi(x, q))=A(\phi(x, q))-b .
$$

Let $h \neq 0$ and $H(t) \neq 0$ denote the so-called auxiliary parameter and auxiliary matrix, respectively. Using the embedding parameter $q \in[0,1]$, we construct a family of equations

$$
(1-q) L\left(\phi(x, q)-x_{0}\right)=h q H(x) N(\phi(x, q))
$$

from (2) and (3) we have

$$
(1-q)\left(\phi(x, q)-x_{0}\right)=h q H(x)(A(\phi(x, q))-b) .
$$

Obviously, at $q=0$ and $q=1$, one has $\phi(x, 0)=x_{0}$ and $A(\phi(x, 1))=b$ respectively. Thus, as $q$ increases from 0 to $1, \phi(x ; q)$ varies continuously from $x_{0}$ to $x$. Such kind of continuous variation is called deformation in topology [16]. We call the family of equations like (4) the zeroth-order deformation equation. Now we define $m$ th-order deformation derivative

$$
x_{m}=\left.\frac{1}{m !} \frac{\partial^{m} \phi(x, q)}{\partial q^{m}}\right|_{q=0},
$$

where $m=1,2,3, \cdots$. Because $\phi(x, q)$ is now a function of the embedding parameter $q$, by Taylors Theorem, we expand $\phi(x ; q)$ in a power series of the embedding parameter $q$ as follows:

$$
\phi(x, q)=\phi(x, 0)+\left.\sum_{m=1}^{+\infty} \frac{1}{m !} \frac{\partial^{m} \phi(x, q)}{\partial q^{m}}\right|_{q=0} q^{m} .
$$

By using (5) we have

$$
\phi(x, q)=x_{0}+\sum_{m=1}^{+\infty} x_{m} q^{m} .
$$

If the series (6) is convergent at $q=1$, then using the relationship $\phi(x, 1)=x$ one has the series solution

$$
x=x_{0}+\sum_{m=1}^{+\infty} x_{m} .
$$

Now we have the so-called $m$ th-order deformation equation

$$
L\left[x_{m}-\chi_{m} x_{m-1}\right]=h H(x) R_{m}\left(\tilde{x}_{m-1}\right),
$$

where

$$
R_{m}\left(\tilde{x}_{m-1}\right)=\left.\frac{1}{(m-1) !} \frac{\partial^{m-1} N(\phi(x, q))}{\partial q^{m-1}}\right|_{q=0}
$$

and

$$
\chi_{m}= \begin{cases}0 & \text { when } m \leq 1 \\ 1 & \text { otherwise }\end{cases}
$$

By using (2) we obtain

$$
\begin{aligned}
& x_{m}-\chi_{m} x_{m-1}=h H(x) R_{m}\left(\vec{x}_{m-1}\right), \\
& x_{m}=\chi_{m} x_{m-1}+h H(x) R_{m}\left(\vec{x}_{m-1}\right) .
\end{aligned}
$$


Also by using (3) and (9) we have

$$
\begin{aligned}
R_{m}\left(\tilde{x}_{m-1}\right) & =\left.\frac{1}{(m-1) !} \frac{\partial^{m-1}(A \phi(x, q)-b)}{\partial q^{m-1}}\right|_{q=0} \\
& =\left.\frac{1}{(m-1) !} \frac{A \partial^{m-1}(\phi(x, q))}{\partial q^{m-1}}\right|_{q=0}-\left.\frac{1}{(m-1) !} \frac{\partial^{m-1}(b)}{\partial q^{m-1}}\right|_{q=0},
\end{aligned}
$$

and then

$$
R_{m}\left(\tilde{x}_{m-1}\right)=A x_{m-1}-b\left(1-\chi_{m}\right) .
$$

Finally by using (11) we obtain

$$
x_{m}=\chi_{m} x_{m-1}+h H(x)\left(A x_{m-1}-b\left(1-\chi_{m}\right)\right) .
$$

Now with the initial guess $x_{0}=0$ and $h=-1$ we have

$$
\begin{aligned}
x_{1} & =H(x) b, \\
x_{2} & =x_{1}-H(x) A x_{1}=(I-H(x) A) x_{1}=(I-H(x) A) H(x) b, \\
x_{3} & =x_{2}-H(x) A x_{2}=(I-H(x) A) x_{2}=(I-H(x) A)^{2} H(x) b, \\
& \vdots \\
x_{n} & =x_{n-1}-H(x) A x_{n-1}=(I-H(x) A) x_{n-1}=(I-H(x) A)^{n-1} H(x) b,
\end{aligned}
$$

hence, by substituting (13) in (7) we obtain

$$
\begin{aligned}
x & =x_{1}+x_{2}+x_{3}+\cdots+x_{n}+\cdots \\
= & H(x) b+(I-H(x) A) H(x) b+(I-H(x) A)^{2} H(x) b+\cdots \\
& +(I-H(x) A)^{n-1} H(x) b+\cdots,
\end{aligned}
$$

and by factor of $H(x) b$ we have

$$
x=\left[I+(I-H(x) A)+(I-H(x) A)^{2}+\cdots+(I-H(x) A)^{n-1}+\cdots\right] H(x) b .
$$

Now we have to prove the convergence of (15).

Theorem 1. The sequence $x^{[m]}=\left[\sum_{k=0}^{m}[I-H(x) A]^{k}\right] H(x) b$, is a Cauchy sequence if

$$
\|I-H(x) A\|<1 .
$$

Proof: Following ([2], Theorem 1) we have to show that

$$
\lim _{m \rightarrow \infty}\left\|x^{[m+p]}-x^{[m]}\right\|=0
$$

Now considering

$$
x^{[m+p]}-x^{[m]}=\left[\sum_{k=1}^{p}[I-H(x) A]^{m+k}\right] H(x) b,
$$

then

$$
\left\|x^{[m+p]}-x^{[m]}\right\| \leq\|H(x) b\| \sum_{k=1}^{p}\|I-H(x) A\|^{m+k}
$$

let $\gamma=\|I-H(x) A\|$ then 


$$
\left\|x^{[m+p]}-x^{[m]}\right\| \leq\|H(x) b\| \gamma^{m} \sum_{k=1}^{p} \gamma^{k} \leq\|H(x) b\| \gamma^{m}\left(\frac{\gamma^{p}-1}{\gamma-1}\right),
$$

so we have

$$
\lim _{m \rightarrow \infty}\left\|x^{[m+p]}-x^{[m]}\right\| \leq\left(\frac{\gamma^{p}-1}{\gamma-1}\right)\|H(x) b\|\left(\lim _{m \rightarrow \infty} \gamma^{m}\right),
$$

since $\gamma<1$ then we obtain

$$
\lim _{m \rightarrow \infty}\left\|x^{[m+p]}-x^{[m]}\right\|=0
$$

which completes the proof.

\section{Main Results}

In this section For solving the linear system (1) we apply different $H(x)$ and the convergence of the method is checked. At first assume that $\mathrm{A}$ is a nonsingular diagonally dominate matrix and $a_{i i} \neq 0, i=1,2, \cdots, n$. Dividing (1) by $a_{i i}$ and without loss of generality we can obtain

$$
B x=d .
$$

where $B=\left[b_{i, j}\right]$, such that

$$
b_{i, j}= \begin{cases}1 & \text { for } i=j, i=1,2, \cdots, n \\ \frac{a_{i, j}}{a_{i, i}} & \text { for } i \neq j, i, j=1,2, \cdots, n,\end{cases}
$$

and

$$
d_{i}=\frac{b_{i}}{a_{i, i}} \quad i=1,2, \cdots, n .
$$

Now we apply different $H(x)$ and the convergence of the method is tested.

1) we propose $H(x)=I+S$ with

$$
S=\left(s_{i j}\right)=\left\{\begin{array}{lc}
-b_{i i+1} & \text { for } i=1,2, \cdots, n-1, j=i+1 \\
0 & \text { otherwise }
\end{array}\right.
$$

and show that

$$
\|I-H(x) B\|<1 .
$$

Theorem 2. If $A$ is diagonally dominated and $B=\left[b_{i, j}\right]$, where $b_{i, j}$ is defined in (17) then

$$
\|I-H(x) B\|_{\infty}<1
$$

Proof: By direct calculation we have

$$
I-H(x) B=\left[\begin{array}{ccccc}
b_{12} b_{21} & 0 & -b_{13}+b_{12} b_{23} & \ldots & -b_{1 n}+b_{12} b_{2 n} \\
-b_{21}+b_{23} b_{31} & b_{23} b_{32} & 0 & \ldots & -b_{1 n}+b_{12} b_{2 n} \\
\vdots & \vdots & \ddots & \ddots & \vdots \\
-b_{n-1,1}+b_{n-1, n} b_{n, 1} & -b_{n-1,2}+b_{n-1, n} b_{n, 2} & \cdots & b_{n-1, n} b_{n, n-1} & 0 \\
-b_{n, 1} & -b_{n, 2} & \cdots & -b_{n, n-1} & 0
\end{array}\right]
$$

and first row is satisfied:

$$
\begin{aligned}
& \left|b_{12} b_{21}\right|+\left|-b_{13}+b_{12} b_{23}\right|+\left|-b_{14}+b_{12} b_{24}\right|+\cdots+\left|-b_{1 n}+b_{12} b_{2 n}\right| \\
& \leq\left|b_{12}\right|\left|b_{21}\right|+\left|b_{13}\right|+\left|b_{12}\right|\left|b_{23}\right|+\left|b_{14}\right|+\left|b_{12}\right|\left|b_{24}\right|+\cdots+\left|b_{1 n}\right|+\left|b_{12}\right|\left|b_{2 n}\right| \\
& =\left|b_{12}\right|\left(\left|b_{21}\right|+\left|b_{23}\right|+\left|b_{24}\right|+\cdots+\left|b_{2 n}\right|\right)+\left(\left|b_{13}\right|+\left|b_{14}\right|+\cdots+\left|b_{1 n}\right|\right) .
\end{aligned}
$$


Since $A$ is diagonally dominated, $B$ is diagonally dominated and we have

$$
\left|b_{21}\right|+\left|b_{23}\right|+\left|b_{24}\right|+\cdots+\left|b_{2 n}\right| \leq 1 \text {. }
$$

Now by using (19) we obtain

$$
\begin{aligned}
& \left|b_{12}\right|(\underbrace{\left|b_{21}\right|+\left|b_{23}\right|+\left|b_{24}\right|+\cdots+\left|b_{2 n}\right|}_{\leq 1})+\left(\left|b_{13}\right|+\left|b_{14}\right|+\cdots+\left|b_{1 n}\right|\right) \\
& \leq\left|b_{12}\right|+\left|b_{13}\right|+\left|b_{14}\right|+\cdots+\left|b_{1 n}\right| \leq 1
\end{aligned}
$$

This relation satisfis for other rows also and

$$
\|I-H(x) B\|_{\infty}<1
$$

2) We propose $H(x)=I+R$ with

$$
R=\left\{\begin{array}{lc}
-b_{n j} & \text { for } j=1,2, \cdots, n-1 \\
0 & \text { otherwise }
\end{array}\right.
$$

and show that

$$
\|I-H(x) B\|<1 .
$$

Theorem 3. If $A$ is diagonally dominated and $B=\left[b_{i, j}\right]$, where $b_{i, j}$ is defined in (17) then

$$
\|I-H(x) B\|_{\infty}<1
$$

Proof: Following Theorem (2)

$$
I-H(X) B=\left[\begin{array}{ccccc}
0 & -b_{12} & -b_{13} & \cdots & -b_{1 n} \\
-b_{21} & 0 & -b_{23} & \cdots & -b_{2 n} \\
\vdots & \vdots & \ddots & \ddots & \vdots \\
-b_{n-1,1} & -b_{n-1,2} & \cdots & 0 & -b_{n-1, n} \\
R_{n 1} & R_{n 2} & \cdots & R_{n, n-1} & R_{n, n}
\end{array}\right]
$$

such that

$$
\begin{aligned}
& R_{n 1}=b_{n, 2} b_{21}+b_{n, 3} b_{31}+\cdots+b_{n, n-1} b_{n-1,1}, \\
& R_{n 2}=b_{n, 1} b_{12}+b_{n, 3} b_{32}+\cdots+b_{n, n-1} b_{n-1,2}, \\
& \vdots \\
& R_{n, n-1}=b_{n, 1} b_{1, n-1}+b_{n, 2} b_{2, n-1}+\cdots+b_{n, n-2} b_{n-2, n-1}, \\
& R_{n, n}=b_{n, 1} b_{1, n}+b_{n, 2} b_{2, n}+\cdots+b_{n, n-1} b_{n-1, n}
\end{aligned}
$$

and last row is satisfied:

$$
\begin{aligned}
& \left|b_{n, 2} b_{21}+b_{n, 3} b_{31}+\cdots+b_{n, n-1} b_{n-1,1}\right|+\left|b_{n, 1} b_{12}+b_{n, 3} b_{32}+\cdots+b_{n, n-1} b_{n-1,2}\right|+\cdots \\
& +\left|b_{n, 1} b_{1, n-1}+b_{n, 2} b_{2, n-1}+\cdots+b_{n, n-2} b_{n-2, n-1}\right|+\left|b_{n, 1} b_{1, n}+b_{n, 2} b_{2, n}+\cdots+b_{n, n-1} b_{n-1, n}\right| \\
& \leq\left|b_{n, 1}\right|(\underbrace{\left|b_{12}\right|+\left|b_{13}\right|+\cdots+\left|b_{1, n}\right|}_{\leq 1})+\left|b_{n, 2}\right|(\underbrace{\left|b_{21}\right|+\left|b_{23}\right|+\cdots+\left|b_{2, n}\right|}_{\leq 1}) \\
& \quad+\left|b_{n, 3}\right|(\underbrace{\left|b_{31}\right|+\left|b_{32}\right|+\cdots+\left|b_{3, n}\right|}_{\leq 1})+\cdots+\left|b_{n, n-1}\right|(\underbrace{\left|b_{n-1,1}\right|+\left|b_{n-1,2}\right|+\cdots\left|b_{n-1, n}\right|}_{\leq 1}) \\
& \leq\left|b_{n, 1}\right|+\left|b_{n, 2}\right|+\cdots\left|b_{n, n-1}\right| \leq 1
\end{aligned}
$$

This relation satisfis for other rows also 


$$
\|I-H(x) B\|_{\infty}<1
$$

3) We propose $H(x)=I+S+R$ such that $S$ and $R$ was explained in (18) and (20) respectively and show that

$$
\|I-H(x) B\|<1
$$

Theorem 4. If $A$ is diagonally dominated and $B=\left[b_{i, j}\right]$, where $b_{i, j}$ is defined in (17) then

$$
\|I-H(x) B\|_{\infty}<1
$$

Proof: Similar to proof of Theorems (2) and (3).

4) We propose $H(x)=I+S(m)$ with

$$
\begin{aligned}
& S(m)=\left(S_{i j}\right)= \begin{cases}-b_{i k_{i}} & \text { for } 1 \leq i<n, j>i \\
0 & \text { otherwise, }\end{cases} \\
& k_{i}=\left\{\min j\left|\max _{j}\right| a_{i j} \mid\right\}, \quad 1 \leq i<n
\end{aligned}
$$

and show that

$$
\|I-H(x) B\|<1
$$

Theorem 5. If $A$ is diagonally dominated and $B=\left[b_{i, j}\right]$, where $b_{i, j}$ is defined in (17) then

$$
\|I-H(x) B\|_{\infty}<1
$$

Proof: Following Theorem (2) after expanding $I-H(x) B$ according to the first row we have

$$
\begin{aligned}
& \left|b_{1 k_{1}} b_{k_{1} 1}\right|+\left|-b_{12}+b_{1 k_{1}} b_{k_{1} 2}\right|+\left|-b_{13}+b_{1 k_{1}} b_{k_{1} 3}\right|+\cdots+\left|-b_{1 k_{1}-1}+b_{1 k_{1}} b_{k_{1} k_{1}-1}\right| \\
& +0+\left|-b_{1 k_{1}+1}+b_{1 k_{1}} b_{k_{1} k_{1}+1}\right|+\cdots+\left|-b_{1 n}+b_{1 k_{1}} b_{k_{1} n}\right| \\
& \leq\left|b_{1 k_{1}}\right|\left|b_{k_{1} 1}\right|+\left|-b_{12}\right|+\left|b_{1 k_{1}}\right|\left|b_{k_{1} 2}\right|+\left|-b_{13}\right|+\left|b_{1 k_{1}}\right|\left|b_{k_{1} 3}\right|+\cdots+\left|-b_{1 k_{1}-1}\right| \\
& \quad+\left|b_{1 k_{1}}\right|\left|b_{k_{1} k_{1}-1}\right|+\left|-b_{1 k_{1}+1}\right|+\left|b_{1 k_{1}}\right|\left|b_{k_{1} k_{1}+1}\right|+\cdots+\left|-b_{1 n}\right|+\left|b_{1 k_{1}}\right|\left|b_{k_{1} n}\right| \\
& =\left|b_{1 k_{1}}\right| \mid \underbrace{\left.\left|b_{k_{1} 1}\right|+\left|b_{k_{1} 2}\right|+\cdots+\left|b_{k_{1} k_{1}-1}\right|+\left|b_{k_{1} k_{1}+1}\right|+\cdots+\left|b_{k_{1} n}\right|\right)}_{\leq 1} \\
& \quad+\left(\left|-b_{12}\right|+\left|-b_{13}\right|+\cdots+\left|-b_{1 k_{1}-1}\right|+\left|-b_{1 k_{1}+1}\right|+\cdots+\left|-b_{1 n}\right|\right) \\
& \leq\left|b_{1 k_{1}}\right|+\left|-b_{12}\right|+\left|-b_{13}\right|+\cdots+\left|-b_{1 k_{1}-1}\right|+\left|-b_{1 k_{1}+1}\right|+\cdots+\left|-b_{1 n}\right| \leq 1 .
\end{aligned}
$$

This relation satisfis for other rows also

$$
\|I-H(x) B\|_{\infty}<1 \text {. }
$$

5) We propose $H(x)=I+S(m)+R$ such that $S(m)$ and $R$ was explained in (21) and (20) respectively and show that

$$
\|I-H(x) B\|<1 .
$$

Theorem 6. If $A$ is diagonally dominated and $B=\left[b_{i, j}\right]$, where $b_{i, j}$ is defined in (17) then

$$
\|I-H(x) B\|_{\infty}<1
$$

Proof: Similar to proof of Theorems (3) and (5).

6) We propose $H(x)=I-U$ such that $U$ is the strictly upper triangular part of $A$ and show that 


$$
\|I-H(x) B\|<1 .
$$

Theorem 7. If $A$ is diagonally dominated and $B=\left[b_{i, j}\right]$, where $b_{i, j}$ is defined in (17) then

$$
\|I-H(x) B\|_{\infty}<1
$$

Proof: Following Theorem (2) after expanding $I-H(x) B$ according to the first row we have

$$
\begin{aligned}
& \left|b_{12} b_{21}+b_{13} b_{31}+\cdots+b_{1 n} b_{n 1}\right|+\left|b_{13} b_{32}+b_{14} b_{42}+\cdots+b_{1 n} b_{n 2}\right| \\
& +\left|b_{12} b_{23}+b_{14} b_{43}+\cdots+b_{1 n} b_{n 3}\right|+\cdots+\left|b_{12} b_{2 n}+b_{13} b_{3 n}+\cdots+b_{1 n-1} b_{n-1, n}\right| \\
\leq & \left|b_{12}\right|\left|b_{21}\right|+\left|b_{13}\right|\left|b_{31}\right|+\cdots+\left|b_{1 n}\right|\left|b_{n 1}\right|+\left|b_{13}\right|\left|b_{32}\right|+\left|b_{14}\right|\left|b_{42}\right|+\cdots+\left|b_{1 n}\right|\left|b_{n 2}\right| \\
& +\left|b_{12}\right|\left|b_{23}\right|+\left|b_{14}\right|\left|b_{43}\right|+\cdots+\left|b_{1 n}\right|\left|b_{n 3}\right|+\cdots+\left|b_{12}\right|\left|b_{2 n}\right|+\left|b_{13}\right|\left|b_{3 n}\right|+\cdots+\left|b_{1 n-1}\right|\left|b_{n-1, n}\right| \\
= & \left|b_{12}\right|(\underbrace{\left|b_{21}\right|+\left|b_{23}\right|+\cdots+\left|b_{2 n}\right|}_{\leq 1})+\left|b_{13}\right|(\underbrace{\left|b_{31}\right|+\left|b_{32}\right|+\left|b_{34}\right|+\cdots+\left|b_{3 n}\right|}_{\leq 1})+\cdots \\
& +\left|b_{1 n}\right|(\underbrace{\left|b_{n 1}\right|+\left|b_{n 2}\right|+\cdots+\left|b_{n, n-1}\right|}_{\leq 1}) \leq \underbrace{\left|b_{12}\right|+\left|b_{13}\right|+\cdots+\left|b_{1 n}\right| \leq 1}_{\leq 1}
\end{aligned}
$$

This relation satisfis for other rows also

$$
\|I-H(x) B\|_{\infty}<1
$$

7) We propose $H(x)=I-U+R$ such that $U$ is the strictly upper triangular part of $A$ and $R$ was explained in (20) and show that

$$
\|I-H(x) B\|<1 .
$$

Theorem 8. If $A$ is diagonally dominated and $B=\left[b_{i, j}\right]$, where $b_{i, j}$ is defined in (17) then

$$
\|I-H(x) B\|_{\infty}<1
$$

Proof: Similar to proof of Theorems (3) and (7).

Now in the next section we apply $H(x)$ for solving numerical examples.

\section{Numerical Results}

In this section, we present some numerical examples to apply HAM and HPM methods for solving linear system. We used of Matlab 2013 for numerical results.

Example 1. Consider the linear system $A x=b$, that $A=\left[\begin{array}{ccc}4 & 1 & -1 \\ -1 & 6 & 2 \\ 0 & 1 & -3\end{array}\right], \quad b=\left[\begin{array}{l}7 \\ 9 \\ 5\end{array}\right]$ and the exact solution is $x=\left[\begin{array}{c}1 \\ 2 \\ -1\end{array}\right]$.

Table 1 shows the iteration number,error,spectral radius of iteration matrix and computation time.

According to Table 1 we obtain the desirable result for solving this system by seven iterations with HAM and $H(x)=I-U+R$ while by HPM method we used of fourteen iteration.

In this example the matrices $S$ and $S(m)$ are same and the results are same too.

Example 2. In this example we apply HAM method for solving the linear system

$$
A x=b
$$

where $A$ is a $1000 \times 1000$ matrix, $b$ is a $1000 \times 1$ vector that its components are sum of the row components of the corresponding matrix and the exact solution is $\left[\begin{array}{cccc}1 & 1 & \cdots & 1\end{array}\right]^{\mathrm{T}}$. The numerical results are in Table 2 . 
Table 1. Camparision between HPM and HAM for $3 \times 3$ system.

\begin{tabular}{lcccc}
\multicolumn{1}{c}{ Method } & Iteration & Error & Spectral radius & Times (s) \\
\hline $\operatorname{HAM}(H(x)=I)(\mathrm{HPM})$ & 14 & $10^{-5}$ & 0.4004 & 0.013 \\
$\operatorname{HAM}(H(x)=I+S)$ & 11 & $10^{-5}$ & 0.3057 & 0.010 \\
$\operatorname{HAM}(H(x)=I+R)$ & 9 & $10^{-5}$ & 0.2168 & 0.010 \\
$\operatorname{HAM}(H(x)=I+R+S)$ & 8 & $10^{-5}$ & 0.1806 & 0.014 \\
$\operatorname{HAM}(H(x)=I+S(m))$ & 11 & $10^{-5}$ & 0.2918 & 0.010 \\
$\operatorname{HAM}(H(x)=I+S(m)+R)$ & 8 & $10^{-5}$ & 0.1806 & 0.014 \\
$\operatorname{HAM}(H(x)=I-U)$ & 8 & $10^{-5}$ & 0.2918 & 0.011 \\
$\operatorname{HAM}(H(x)=I-U+R)$ & 7 & $10^{-5}$ & 0.1667 & 0.011 \\
\hline
\end{tabular}

Table 2. Camparision between HPM and HAM for $1000 \times 1000$ system.

\begin{tabular}{lcccc}
\hline \multicolumn{1}{c}{ Method } & Iteration & Error & Spectral radius & Times (s) \\
\hline $\operatorname{HAM}(H(x)=I)(\mathrm{HPM})$ & 36 & $10^{-4}$ & 0.5258 & 16.378 \\
$\operatorname{HAM}(H(x)=I+S)$ & 32 & $10^{-4}$ & 0.5167 & 19.141 \\
$\operatorname{HAM}(H(x)=I+R)$ & 32 & $10^{-4}$ & 0.5157 & 17.176 \\
$\operatorname{HAM}(H(x)=I+R+S)$ & 32 & $10^{-4}$ & 0.5169 & 18.010 \\
$\operatorname{HAM}(H(x)=I+S(m))$ & 32 & $10^{-4}$ & 0.5162 & 20.112 \\
$\operatorname{HAM}(H(x)=I+S(m)+R)$ & 32 & $10^{-4}$ & 0.5261 & 19.200 \\
$\operatorname{HAM}(H(x)=I-U)$ & 25 & $10^{-4}$ & 0.4143 & 11.731 \\
$\operatorname{HAM}(H(x)=I-U+R)$ & 20 & $10^{-4}$ & 0.4092 & 9.175 \\
\hline
\end{tabular}

\section{Conclusion}

From the numerical results, we have seen that the HAM method with different $H(x)$ produces a spectral radius smaller than the HPM and with the less iteration we obtain the desirable result.

\section{Acknowledgements}

We thank Islamic Azad University for support researcher plan entitled: "Combination of Iterative methods and semi analytic methods for solving linear systems" and the Editor and the referee for their comments.

\section{References}

[1] Nazari, A.M. and Zia Borujeni, S. (2012) A Modified Precondition in the Gauss-Seidel Method. Advances in Linear Algebra \& Matrix Theory, 1, 31-37. http://dx.doi.org/10.4236/alamt.2012.23005

[2] Keramati, B. (2009) An Approach to the Solution of Linear System of Equations by He's Homotopy Perturbation Method. Chaos, Solitons and Fractals, 41, 152-156. http://dx.doi.org/10.1016/j.chaos.2007.11.020

[3] Liu, H.K. (2011) Application of Homotopy Perturbation Methods for Solving Systems of Linear Equations. Applied Mathematics and Computation, 217, 5259-5264. http://dx.doi.org/10.1016/j.amc.2010.11.024

[4] Liao, S.J. (1992) The Proposed Homotopy Analysis Technique for the Solution of Nonlinear Problems. Ph.D. Thesis, Shanghai Jiao Tong University, Shanghai.

[5] Morimoto, M., Harada, K., Sakakihara, M. and Sawami, H. (2004) The Gauss-Seidel Iterative Method with the Preconditioning Matrix. $\left(I+S+S_{m}\right)$. Japan Journal of Industrial and Applied Mathematics, 21, 25-34.

http://dx.doi.org/10.1007/BF03167430 
[6] Niki, H., Kohno, T. and Morimoto, M. (2008) The Preconditioned Gauss-Seidel Method Faster than the SOR Method. Journal of Computational and Applied Mathematics, 218, 59-71. http://dx.doi.org/10.1016/j.cam.2007.07.002

[7] Niki, H., Kohno, T. and Abe, K. (2009) An Extended GS Method for Dense Linear System. Journal of Computational and Applied Mathematics, 231, 177-186. http://dx.doi.org/10.1016/j.cam.2009.02.005

[8] Yusefoglu, E. (2009) An Improvement to Homotopy Perturbation Method for Solving System of Linear Equations. Computers \& Mathematics with Applications, 58, 2231-2235. http://dx.doi.org/10.1016/j.camwa.2009.03.010

[9] Yuan, J.Y. and Zontini, D.D. (2012) Comparison Theorems of Preconditioned Gauss-Seidel Methods for M-Matrices. Applied Mathematics and Computation, 219, 1947-1957. http://dx.doi.org/10.1016/j.amc.2012.08.037

[10] Gunawardena, A.D., Jain, S.K. and Snyder, L. (1991) Modified Iterative Method for Consistent Linear Systems. Linear Algebra and Its Applications, 154-156, 123-143. http://dx.doi.org/10.1016/0024-3795(91)90376-8

[11] Kohno, T. and Niki, H. (2009) A Note on the Preconditioner Pm $=(I+S m)$. Journal of Computational and Applied Mathematics, 225, 316-319. http://dx.doi.org/10.1016/j.cam.2008.07.042

[12] He, J.H. (2003) Homotopy Perturbation Method: A New Non-Linear Analytical Technique. Applied Mathematics and Computation, 135, 73-79. http://dx.doi.org/10.1016/S0096-3003(01)00312-5

[13] Saad, Y. (2003) Iterative Methods for Sparse Linear Systems. SIAM Press, PHiladelphia. http://dx.doi.org/10.1137/1.9780898718003

[14] Liao, S.J. (2003) Beyond Perturbation: Introduction to the Homotopy Analysis Method. Chapman \& Hall/CRC Press, Boca Raton. http://dx.doi.org/10.1201/9780203491164

[15] Liao, S.J. (2009) Notes on the Homotopy Analysis Method: Some Definitions and Theorems. Communications in Nonlinear Science and Numerical Simulation, 14, 983-997. http://dx.doi.org/10.1016/j.cnsns.2008.04.013

[16] Sen, S. (1983) Topology and Geometry for Physicists. Academic Press, Waltham. 